$-25^{\circ} \mathrm{C}$ e processate entro $24 \mathrm{~h}$ per l'estrazione del DNA con il metodo di estrazione di DNA da tessuti High pure PCR Template Preparation Kit ${ }^{\circledR}$ dalla Ditta ROCHE, che prevede digestione con Proteinasi $K^{\circledR}$, seguita da eluizione su colonne High Pure Filter Tube ${ }^{\circledR}$. La PCR sugli eluati è stata eseguita utilizzando la DNA Polimerasi AmpliTaq Gold ${ }^{\circledR}$ della Ditta Applied Biosystem secondo il protocollo di amplificazione della regione spaziatrice $16 \mathrm{~S}-23 \mathrm{~S}$ del genere Mycobacterium indicato dal kit INNO-LiPA MYCOBACTERIA v2 Amp ${ }^{\circledR}$ della Ditta INNOGENETICS. I seguenti cicli termici sono stati eseguiti su termociclatore P.E. Cetus PE2400:

1. Denaturazione $\quad 95^{\circ} \mathrm{C} \quad 1 \mathrm{~min}$

2. Denaturazione $\quad 95^{\circ} \mathrm{C} \quad 30 \mathrm{sec}$

3. Appaiamento $\quad 62^{\circ} \mathrm{C} \quad 30 \mathrm{sec}$

4. Appaiamento $\quad 72^{\circ} \mathrm{C} \quad 30 \mathrm{sec}$

40 volte

5. Hold

$4^{\circ} \mathrm{C}$

$\infty$

L'identificazione degli amplificati è stata effettuata con il metodo INNO-LiPA MYCOBACTERIA v2 ${ }^{\circledR}$ della Ditta INNOGENETICS, basato sul principio della ibridazione inversa: i prodotti dell'amplificazione sono stati biotinilati ed ibridati con sonde oliginucleotidiche immobilizzate su strisce di reazione, corrispondenti a 22 specie di Mycobacterium; altre 2 linee di controllo corrispondono rispettivamente al controllo di reazione del coniugato ed alla presenza del gene comune al genere Mycobacterium. L'ibridazione viene svelata dall'incubazione della striscia in presenza di streptoavidina legata a fosfatasi alcalina e successivamente dall'aggiunta del cromogeno.

Risultati. La presenza di DNA di M. gordonae nel campione è evidenziata dalla corrispondente linea di ibridazione inversa sulla striscia di reazione. (Fig.1)

Discussione. La ricerca tramite PCR di bacilli appartenenti al Genere Mycobacterium in campioni bioptici intestinali, può contribuire a porre la diagnosi differenziale tra $\mathrm{M}$. di Crohn e Tubercolosi intestinale. Nel caso in esame la coltura confermava dopo 21 giorni la presenza di M. gordonae. L'esperienza descritta suggerisce l'utilità di estendere la ricerca di Micobatteri anche a specie non comunemente coinvolte in processi intestinali, ricorrendo a metodi molecolari, altamente specifici e più sensibili rispetto ai metodi tradizionali.

Figura 1

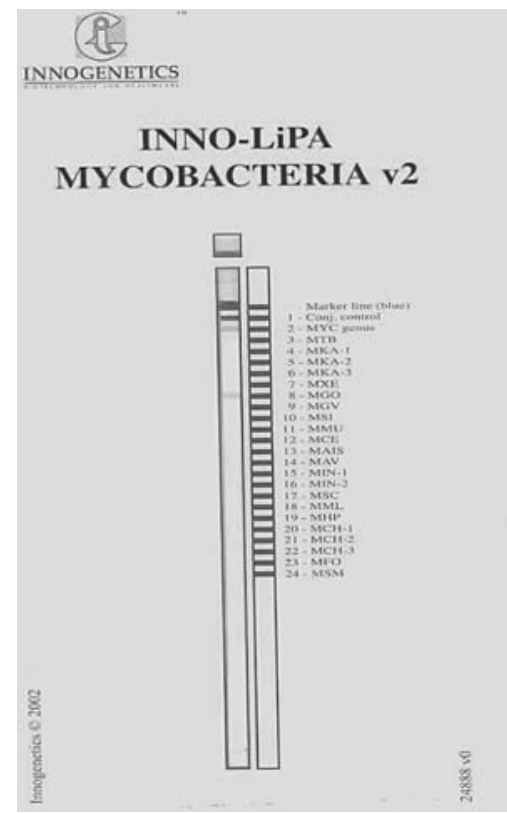

190

\section{GENOTIPI DI VIRUS C IN UN CAMPIONE DI PAZIENTI AFFERENTI ALLA U.O. SEMPLICE DI MICROBIOLOGIA, P.O. CASTROVILLARI}

Venditti W., Bonifati A., Capano A., Di Vasto M., Maltese R., Vicchio T.

Unità Operativa di Microbiologia, P.O. Castrovillari (CS),
Azienda Sanitaria N. 2

Introduzione. L'infezione da virus dell'epatite $\mathrm{C}$ rappresenta attualmente uno dei più importanti problemi di sanità pubblica a livello mondiale.

I virus che causano l'epatite $\mathrm{C}$ costituiscono un gruppo che presenta sottotipi estremamente variabili. Lo studio del genotipo del virus HCV rappresenta un momento importante nella definizione epidemiologica e clinica dell'infezione. Il genotipo identifica la virulenza del ceppo infettante e consente di individuare categorie differenti di pazienti a cui applicare protocolli di trattamento antivirale differenziati. In Italia ed in Europa vi è una netta prevalenza del genotipo 1 , ed in particolare del genotipo $1 \mathrm{~b}$.

Materiali e metodi. Nell'arco temporale 2003-2005 sono stati tipizzati i genotipi HCV di 211 pazienti, di cui 71 provenienti dal SERT e 140 ambulatoriali. L'RNA virale veniva estratto da campioni di siero la cui positività è stata rivelata mediante RT- PCR (Cobas Amplicor HCV, Roche) e genotipizzato mediante tecnica di ibridazione inversa su supporto solido (Versant HCV genotipo LiPA, Bayer).

Risultati. Il genotipo $1 \mathrm{~b}$ è stato riscontrato nel $41 \%$ dei pazienti ambulatoriali e nel $6 \%$ dei pazienti provenienti dal SERT; il genotipo 1a rispettivamente nel 16 e $19 \%$; il $2 \mathrm{a}$ / 2 c è stato riscontrato nel 5 e $43 \%$; il 2 a nel 10 e $1 \%$; il 3 a nel 26 e $25 \%$; il 4 nel 2 e $7 \%$.

Conclusioni. Il genotipo $1 \mathrm{~b}$ è risultato essere, come ci si attendeva, il più frequente tra i pazienti ambulatoriali, i genotipi $2 \mathrm{a}$ e 4 i meno frequenti; nei pazienti afferenti al SERT è risultato essere più frequente il genotipo $2 \mathrm{a} / 2 \mathrm{c}$. In particolare lo studio del genotipo di HCV in questa ultima popolazione di pazienti, ad alto rischio di trasmissione del virus, rappresenta un utile marcatore di diffusione del virus, offrendo utili informazioni sulla modalità di selezione e circolazione dei genotipi virali.

\section{1}

\section{HPV: CONDIZIONE NECESSARIA MA NON SUFFICIENTE NELL'EZIOLOGIA DEL CANCRO DELLA CERVICE}

Venditti W.', Bonifati A.', Di Vasto M.', Stigliano C.M. ${ }^{3}$, Caruso W. ${ }^{2}$, Fabrizio $A^{3}$., Schifino $F^{3}$.

U.O. semplice Microbiologia

U.O. Anatomia Patologica

3U.O. Ginecologia Preventiva, P.O. Castrovillari (CS), Azienda Sanitaria N. 2

Introduzione. I papillomavirus sono virus a DNA a simmetria icosaedrica, mancanti di rivestimento pericapsidico. Dati biologici ed epidemiologici hanno portato a suddividere gli 
HPV in alto, medio e basso rischio oncogenico in base al rischio più o meno significativo nell'indurre la trasformazione neoplastica. Gli HPV ad alto rischio rappresentano la principale causa dell'insorgenza di tumori della cervice uterina. L'infezione da HPV ad alto rischio non conduce all'immediato sviluppo di neoplasie invasive in quanto nella maggior parte dei casi l'infezione si risolve da sola, in altri il virus rimane latente per lunghi periodi di tempo, fino anche 20-50 anni. Il picco di incidenza dell'infezione genitale nelle donne si evidenzia nelle fasce di età intorno ai 20 anni, invece la malattia neoplastica necessita di una fase di latenza e raggiunge la sua massima incidenza nelle donne con oltre $i$ 40 anni di età. È evidente che nella eziopatogenesi tumorale sia necessaria la persistenza dell'infezione virale. Tuttavia come un'infezione produttiva sia in grado di diventare a lunga permanenza e/o quali fattori possano dare luogo a lesioni cervicali reversibili dipende verosimilmente dalle situazioni fisiologiche ed immunologiche di ciascuna donna. Metodi. Il nostro studio effettuato in circa sei mesi di lavoro su pazienti afferenti al centro di Ginecologia Preventiva ${ }^{2}$ età compresa tra i $16-75$ anni sottoposte a pap-test e a ricerca dell'HPV-DNA.

Il test utilizzato per il riconoscimento dell'infezione da HPV si basa sulla tecnica del branched- DNA con una amplificazione finale del segnale, il test non distingue i sottotipi di papillomi implicati, ma stratifica il rischio di sviluppo di cancro della cervice distinguendo gli HPV in alto e basso rischio oncogeno, fornisce inoltre informazioni circa la carica virale infettante.

Risultati. Su un totale di 305 pazienti il riscontro dell'infezione da HPV è stato di 76 positivi pari al $25 \%$ ed era correlato alle seguenti alterazioni citologiche:

PAP-NEGATIVO 40 pari al

ASCUS 8 pari al $10,5 \%$

L-SIL 13 pari al $17,1 \%$

$\mathrm{H}$-SIL 12 pari al $15,7 \%$ di cui 5 CIS

Conclusioni. La presenza di HPV alto rischio con pap-test negativo si è riscontrato in pazienti di età compresa tra 18 e 35 anni. HPV alto rischi con ascus ed 1-sil, in pazienti di età compresa tra 35 e 42 anni.

HPV alto rischio con h-sil e ca microinvasivo in pazienti con età superiore ai 43 anni.

${ }^{2}$ Trattasi di centro di riferimento regionale di secondo livello

\section{2}

\section{VALUTAZIONE DELLA PERSISTENZA DI DIVERSI GENOTIPI DI HPV NEL FOLLOW-UP DI PAZIENTI TRATTATE PER LESIONI CERVICALI DI ALTO GRADO}

Venturoli S.', Ambretti S.', Cricca M.',2, Gallinella G.', Manaresi E.', Bonvicini F.', Filippone C.', Musiani M.', Zerbini M.'

\section{'D.M.C.S.S - Divisione di Microbiologia, Università di Bologna, ${ }^{2}$ C.R.R.E.M. - Azienda Ospedaliera S.Orsola Malpighi; Via Massarenti 9, 40138 Bologna}

Introduzione. Molti studi di follow up su pazienti sottoposte a trattamento di conizzazione per lesioni cervicali di alto grado (CIN2/3, carcinoma invasivo) hanno dimostrato una elevata persistenza degli HPV ad alto rischio oncogeno (HR-
HPV) anche nei casi in cui la lesione è stata completamente rimossa. La persistenza del virus aumenta il rischio di recidiva della lesione.

Scopo del nostro studio è stato valutare la persistenza di HPV dopo conizzazione e la percentuale di clearance dei diversi genotipi. A tale scopo abbiamo impiegato il metodo di PCR combinato a genotipizzazione [Line Blot Assay-Roche (LBA)] nell'analisi di 35 pazienti con lesioni di alto grado.

Metodi. Trentacinque donne sottoposte a conizzazione per lesioni di alto grado e risultate positive per HR-HPV in Hybrid Capture II sono state selezionate per il nostro studio. Di tutte le pazienti è stato analizzato, impiegando il saggio di amplificazione e genotipizzazione PCR-LBA, il prelievo al momento del trattamento e un prelievo a 3-6 mesi. I risultati ottenuti con il metodo di tipizzazione LBA sono stati confrontati con quelli ottenuti con un metodo di tipizzazione ELISA home-made.

Di tutte le pazienti è stato osservato l'istologico su cono e lo stato dei margini al fine di valutare la corretta rimozione della lesione. Inoltre tali pazienti hanno mostrato un Pap test normale nel controllo a 3-6 mesi.

Risultati. Al momento del trattamento, i genotipi identificati con maggiore frequenza sono stati HPV 16 (47\%), HPV31 (12\%) e HPV33 (12\%), mentre il $25 \%$ delle pazienti analizzate ha mostrato un'infezione mista. Nel $44 \%$ dei prelievi eseguiti a 3-6 mesi dal trattamento si è evidenziata una persistenza dell'infezione da HR HPV. La persistenza virale è risultata differente a seconda del genotipo, infatti le pazienti con infezione da HPV16 hanno evidenziato una clearance del $67 \%$, contro il $47 \%$ di quelle interessate da altri genotipi ad alto rischio oncogeno (31-33-52-58).

Conclusioni. L'impiego di tecniche per la genotipizzazione di un ampio spettro di HPV, quali il metodo Line Blot Assay (Roche), permette di valutare la persistenza virale dopo trattamento chirurgico per lesioni di alto grado e di correlare la clearance virale ai diversi genotipi di HPV. La persistenza dell'infezione da HR-HPV in pazienti trattate per lesioni cervicali di alto grado e con citologia negativa rappresenta un importante fattore predittivo del rischio di recidiva.

\section{3}

\section{VALUTAZIONE DI UN SISTEMA DIAGNOSTICO AUTOMATIZZATO PER LO STUDIO MOLECOLARE DI INFEZIONI ERPETICHE DEL SISTEMA NERVOSO}

\author{
Verzaro,S., Mancini,C., Nazzari,C., Latte,M.C., Fabri, G., \\ Gaeta, A. \\ Dipartimento di Scienze di Sanità Pubblica, \\ Università degli Studi “La Sapienza", P.le A. Moro 5, 00185 Roma
}

Introduzione. I virus erpetici svolgono un ruolo importante nella eziopatogenesi di varie sindromi infettive a carico del sistema nervoso. Essi sono ritenuti responsabili sia di patologie acute del sistema nervoso centrale con liquor limpido, o meningoencefaliti, sia di sindromi post-infettive ; inoltre il Cytomegalovirus e l'Herpes virus 6 sono stati correlati a patologie del sistema nervoso periferico quali la Sindrome di Guillain - Barrè.

Negli ultimi anni i notevoli progressi ottenuti nella diagnosi virologica hanno rivoluzionato l'approccio clinico verso il paziente neurologico grazie all'impiego di protocolli diagno- 